\title{
Vacuum instability in time-dependent electric fields: New example of an exactly solvable case
}

\author{
A. I. Breev $\odot^{1, *}$ S. P. Gavrilov $\odot^{1,2, \dagger}$ D. M. Gitman,,$^{1,3,4, \sharp}$ and A. A. Shishmarev $\circledast^{1,5, \S}$ \\ ${ }^{1}$ Department of Physics, Tomsk State University, Lenin avenue 36, 634050 Tomsk, Russia \\ ${ }^{2}$ Herzen State Pedagogical University of Russia, Moyka embankment 48, 191186 St. Petersburg, Russia \\ ${ }^{3}$ P.N. Lebedev Physical Institute, 53 Leninskiy ave., 119991 Moscow, Russia \\ ${ }^{4}$ Institute of Physics, University of São Paulo, Rua do Matão, 1371, CEP 05508-090, São Paulo, SP, Brazil \\ ${ }^{5}$ Institute of High Current Electronics, SB RAS, Akademichesky avenue 2/3, 634055 Tomsk, Russia
}

(Received 29 June 2021; revised 17 September 2021; accepted 18 September 2021; published 8 October 2021)

A new exactly solvable case in strong-field quantum electrodynamics with a time-dependent external electric field is presented. The corresponding field is given by one component of the electromagnetic vector potential, which is the analytic function $A_{x}(t)=\sigma E_{0}[1+\exp (t / \sigma)]^{-1 / 2}$, where $\sigma$ is a timescale parameter. In contrast to Sauter-like electric field, this field is asymmetric with respect to the time instant, where it reaches its maximum value, that is why we call it the analytic asymmetric electric field. We managed to exactly solve the Dirac equation with such a field, which made it possible to calculate characteristics of the corresponding vacuum instability nonperturbatively. We construct the so-called in- and out-solutions and with their help calculate mean differential and total numbers of created charged particles, probability of the vacuum to remain a vacuum, vacuum mean values of current density and energy-momentum tensor of the particles. We study the vacuum instability in regimes of rapidly and slowly changing analytic asymmetric electric field, and compare the obtained results with corresponding ones obtained earlier for the case of the symmetric Sauter-like electric field. We also compare exact results in the regime of slowly changing field with corresponding results obtained within the slowly varying field approximation proposed by two of the authors in [S. P. Gavrilov and D. M. Gitman, Phys. Rev. D 95, 076013 (2017)], thus demonstrating the effectiveness of such an approximation.

DOI: $10.1103 /$ PhysRevD.104.076008

\section{INTRODUCTION}

Particle creation from the vacuum by strong electromagnetic and gravitational fields is a remarkable effect (sometimes called the Schwinger effect [1]) predicted by quantum field theory $(Q F T)$. A large number of articles, reviews and books are devoted to the history of its theoretical description, possibilities of its observation and applications, see, e.g., Refs. [2-7] and references there. QFT with external backgrounds is, to a certain extent, an appropriate model for theoretical study of the effect. In the framework of such a model, the particle creation is interpreted as a violation of the vacuum stability. Backgrounds (external fields) that violate the vacuum stability are electriclike fields that are

\footnotetext{
*breev@mail.tsu.ru

gavrilovsergeyp@yahoo.com, gavrilovsp@herzen.spb.ru *dmitrygitman@hotmail.com

§a.a.shishmarev@mail.ru
}

Published by the American Physical Society under the terms of the Creative Commons Attribution 4.0 International license. Further distribution of this work must maintain attribution to the author(s) and the published article's title, journal citation, and DOI. Funded by SCOAP ${ }^{3}$. able to produce nonzero work when interacting with charged particles. Creation of charged particles from the vacuum by electriclike fields needs superstrong field magnitudes compared with the Schwinger critical field $E_{\mathrm{c}}=m^{2} c^{3} / e \hbar \simeq 1.3 \times 10^{16} \mathrm{~V} \cdot \mathrm{cm}^{-1}$. Nevertheless, recent progress in laser physics allows one to hope that this effect will be experimentally observed in the near future even in laboratory conditions, see Ref. [8] for the review. Electronhole pair creation from the vacuum (analogue of the electron-positron pair creation) was recently observed in the graphene by its indirect influence on the graphene conductivity [9] (the graphene conductivity modification due to the particle creation was calculated in [10], some other relevant effects were discussed in Ref. [11]). The need to consider strong fields in the above mentioned model leads, in turn, to the need for a nonperturbative consideration of the interaction with external backgrounds and a development of appropriate methods. Depending on the structure of such backgrounds, different approaches for calculating the effect of the vacuum instability in quantum electrodynamics $(Q E D)$ with strong backgrounds (strongfield $Q E D$ in what follows) were elaborated. The most consistent formulation of the particle production problem is 
formulated for time-dependent external electric fields that are switched on and off at infinitely remote times $t \rightarrow \pm \infty$, respectively. A complete nonperturbative with respect to the external background formulation of strong-field $Q E D$ with such external fields was developed in Refs. [5,12]; it is based on the existence of exact solutions of the Dirac equation with time dependent external field (more exactly, complete sets of exact solutions). When such solutions can be found and all the calculations can be done analytically, we refer to these examples as exactly solvable cases. Usually are considered nonstationary homogenous electric fields of a constant direction. Electromagnetic vector potential for such fields can be chosen as a timelike potential step (scalar potential being zero), therefore, below we call fields of this type $t$-steps. We note that strong-field $Q E D$ with harmonically alternating electric fields also belongs to the exactly solvable cases [13-15]. It should be metioned that there exist physically interesting situations where external backgrounds are represented by constant (time-independent) but spatially inhomogeneous fields; see Refs. [16,17] for the review. However, in this article we discuss only problems of the vacuum instability in strongfield $Q E D$ with $t$-steps.

Until now, there are known only few exactly solvable cases in strong-field $Q E D$ with $t$-steps. Those are Sauterlike (or adiabatic or pulse) electric field, $T$-constant electric field (a uniform electric field which effectively acts during a sufficiently large but finite time interval $T$ ), and the so-called peak electric fields (composed of two parts, one increasing and another one decreasing), see e.g., Refs. [18,19] for a review. In this article, we present a new exactly solvable case in strong-field $Q E D$ with $t$-steps. For the generality, the fields are considered in $d=D+1-$ dimensional Minkowski space-time, parametrized by the coordinates $X=(t, \mathbf{r}), \mathbf{r}=\left(x^{1}=x, x^{2}, \ldots, x^{D}\right)$. So far, the effect has been considered in homogeneous fields with constant direction (along one of the axis, usually along the axis $x$ ), growing on the interval $\left(-\infty, t_{\max }\right)$ monotonically from zero to its maximum value $E_{\max }=\left|\mathbf{E}\left(t_{\max }\right)\right|$ at a time instant $t_{\max }$, and then decay monotonically to zero on the interval $\left(t_{\max },+\infty\right)$. Their electromagnetic potentials can be chosen as timelike steps,

$$
\begin{aligned}
A^{0} & =0, \quad \mathbf{A}=\left(A^{1}(t), 0, \ldots, 0\right), \\
A^{1}(t) & =A_{x}(t)=A(t), \quad A(-\infty)>A(+\infty),
\end{aligned}
$$

such that

$$
\begin{aligned}
\mathbf{E}(t) & =\left(E^{1}(t), 0, \ldots, 0\right), \\
E^{1}(t) & =E_{x}(t)=E(t)=-A^{\prime}(t) \geq 0,
\end{aligned}
$$

We note that among the above exactly solvable cases only the external Sauter-like electric field is given by an analytic function,

$$
\begin{aligned}
E(t) & =E_{\max } \cosh ^{-2}\left(t / T_{S}\right), \\
A_{x}(t) & =-T_{S} \tanh \left(t / T_{S}\right), \quad E_{\max }>0 .
\end{aligned}
$$

This field reaches its maximum value at $t=t_{\max }=0$ and is symmetric with respect to the origin. The vacuum instability in the Sauter-like electric field was first studied in Ref. [20] and then many researchers returned to this problem, since in the case under consideration it was convenient to test various approaches, including approximate ones; see, for example, Refs. [18,21] and references therein.

Here we present a new example of exactly solvable case in which the external field is given by the following analytic function:

$$
\begin{aligned}
E(t) & =\frac{E_{0}}{8} \sqrt{1+\exp (t / \sigma)} \cosh ^{-2}(t / 2 \sigma), \quad E_{0}>0, \quad \sigma>0 \\
E_{\max } & =E\left(t_{\max }\right)=3^{-3 / 2} E_{0}, \quad t_{\max }=\sigma \ln 2 \\
A_{x}(t) & =\frac{\sigma E_{0}}{\sqrt{1+\exp (t / \sigma)}} .
\end{aligned}
$$

Assuming $\sigma=T_{S} / 2$, one can compare this field and the Sauter-like electric field. For large negative $t$, both fields behave in a very similar way. In contrast to the Sauter-like electric field the field (4) is asymmetrical with respect to the time instant $t_{\max }$, where it reaches its maximum value. We call this field configuration the analytic asymmetric field.

We stress that vacuum instability problems which can be analytically studied using the exactly solvable cases, may be useful in understanding similar problems in astrophysics, cosmology, and condense matter physics. In particular, the study of the vacuum instability in the Sauter-like and $T$-constant electric fields is instructive for understanding the conductivity in the graphene and Weyl semimetals as was reported in Refs. [10,22-29]. Note that the vacuum instability in $t$-steps has many similarities with the instability in the de Sitter background, as was noted, e.g., in Refs. [30-33] and cited there works. Besides, using the exactly solvable cases one can develop and test new approximation methods for calculating quantum vacuum effects in strong-field $Q F T$.

Finally, we would like to note that the Sauter-like and $T$-constant electric fields are symmetric relative to the point $t=0$. In such fields distributions of created pairs are symmetric with respect of the longitudinal momentum $p_{x}$. The latter symmetry is not inherent in realistic asymmetric fields. The electric field (4) considered in this article is given (similar to Sauter-like field) by an analytic function but is not symmetric. As we will see below, it corresponds to the exactly solvable case of $t$-step electric field, thus allowing an analytical and nonperturbative study of how field asymmetry affects characteristics of the vacuum instability.

All said above was an incentive for us to study the vacuum instability in the analytic asymmetric field (4). 
This study is the subject of this article which is organized as follows. In Sec. II we find exact solutions of the Dirac equation with the analytic asymmetric field (4), in particular, we construct the so-called in- and out-solutions which are the basis for calculating characteristics of the vacuum instability. With their help, in Sec. III, we find nonperturbatively the vacuum-to-vacuum transition probability as well as differential and total mean numbers of created pairs. We compare these characteristics with ones corresponding to other exactly solvable cases. In the same section, we find mean values of the current density and the energy-momentum tensor of created particles. In Sec. IV we study the behavior of obtained physical quantities in the regime of rapidly and slowly varying analytic asymmetric field (4). Having in hands an exact expression for total mean number of created pairs in the regime of slowly varying field, we compare it with an estimate obtained in an universal slowly varying field approximation proposed in Ref. [34], thus demonstrating the effectiveness of the latter. Some final remarks are presented in Sec. V. Useful for us properties of confluent hypergeometric functions are given in Appendix.

\section{IN- AND OUT-SOLUTIONS}

Let us find solutions to the Dirac equation with electric field (4). The Dirac equation in $(d=D+1)$-dimensional Minkowski space-time with such field has the form ${ }^{1}$ :

$$
\begin{aligned}
i \partial_{t} \psi(X) & =H(t) \psi(X), \\
H(t) & =\gamma^{0}\left\{\gamma^{1}\left[-i \partial_{x}+e A_{x}(t)\right]-i \nabla_{\perp} \gamma_{\perp}+m\right\},
\end{aligned}
$$

where $H(t)$ is a one-particle Dirac Hamiltonian, $\psi(X)$ is a $2^{[d / 2]}$-component spinor $([d / 2]$ stands for the integer part of $d / 2), e>0$ is the absolute value of the electron charge, $m$ is the electron mass, and $\gamma^{\mu}$ are $\gamma$-matrices in $d$ dimensions [35]. The index $\perp$ denotes components of the momentum operator that are perpendicular to the electric field.

We seek solutions of Dirac equation in the following form:

$$
\begin{aligned}
\psi_{n}(X) & =\exp (i \mathbf{p r}) \psi_{n}(t), \quad n=(\mathbf{p}, s), \\
\psi_{n}(t) & =\left\{\gamma^{0} i \partial_{t}-\gamma^{1}\left[p_{x}+e A_{x}(t)\right]-\gamma \mathbf{p}_{\perp}+m\right\} \phi_{n}(t),
\end{aligned}
$$

where $\psi_{n}(t)$ and $\phi_{n}(t)$ are time-dependent spinors, $n$ is a complete set of quantum numbers characterizing the solutions. Spin variables can be separated by the substitution:

$$
\begin{aligned}
\phi_{n}(t) & =\varphi_{n}(t) v_{\chi, s}, \quad \chi= \pm 1, \quad s=\left(s_{1}, s_{2}, \ldots, s_{[d / 2]-1}\right), \\
s_{j} & = \pm 1,
\end{aligned}
$$

\footnotetext{
${ }^{1}$ We use the relativistic system of units, $\hbar=c=1$.
}

where $\varphi_{n}(t)$ are some scalar functions and $v_{\chi, s}$ is a set of constant orthonormalized spinors, satisfying the following conditions:

$$
\gamma^{0} \gamma^{1} v_{\chi, s}=\chi v_{\chi, s}, \quad v_{\chi, s}^{\dagger} v_{\chi^{\prime}, s^{\prime}}=\delta_{\chi, \chi^{\prime}} \delta_{s, s^{\prime}} .
$$

Quantum numbers $s$ and $\chi$ describe the spin polarization (if $d \leq 3$ there are no spin degrees of freedom that are described by the quantum numbers $s$ ). The solutions of Dirac equation (6) which differ only by values of $\chi$ are linearly dependent, so it is sufficient to work only with solutions corresponding to one of the values of $\chi$; see Refs. [36] for more details. The scalar functions $\varphi_{n}(t)$ satisfy the following second-order differential equation:

$$
\begin{aligned}
& \left\{\frac{d^{2}}{d t^{2}}+\left[p_{x}+e A_{x}(t)\right]^{2}+\pi_{\perp}^{2}+i \chi e \dot{A}(t)\right\} \varphi_{n}(t)=0, \\
& \pi_{\perp}=\sqrt{\mathbf{p}_{\perp}^{2}+m^{2}} .
\end{aligned}
$$

Now, we transform Eq. (9) to the Heun equation [37,38] of a special form. To this end, we use the ansatz

$$
\begin{aligned}
\varphi_{n}(t) & =(1+z)^{\alpha_{1}}(1-z)^{\alpha_{2}} u_{n}(z), \quad z=\sqrt{1+\exp (t / \sigma)}, \\
\alpha_{1} & =i \tau \sigma \sqrt{\left(p_{x}-e E_{0} \sigma\right)^{2}+\pi_{\perp}^{2}}, \\
\alpha_{2} & =i \tau \sigma \sqrt{\left(p_{x}+e E_{0} \sigma\right)^{2}+\pi_{\perp}^{2}} .
\end{aligned}
$$

Solutions that differ only by the parameter $\tau$ are also linearly dependent. For us it is sufficient to work only with $\tau=+1$. Substituting (10) into (9), we obtain the Heun equation for function $u_{n}(z)$,

$$
\begin{aligned}
\hat{H}_{n} u_{n}(z)= & 0, \\
\hat{H}_{n}= & \frac{d^{2}}{d z^{2}}+\left(-\frac{1}{z}+\frac{1+2 \alpha_{2}}{z-1}+\frac{1+2 \alpha_{1}}{z+1}\right) \frac{d}{d z} \\
& +\frac{z\left[\alpha_{3}^{2}-\left(\alpha_{1}-\alpha_{2}\right)^{2}\right]+\left(\alpha_{1}-\alpha_{2}+\alpha_{3}\right)}{z(z-1)(z+1)}, \\
\alpha_{3}= & -2 i \chi e \sigma^{2} E_{0}, \quad \tau= \pm 1 .
\end{aligned}
$$

Let us represent the functions $u_{n}(z)$ as follows:

$$
\begin{aligned}
u_{n}(z) & =U_{n} \hat{M}_{n} w_{n}\left(\frac{z+1}{2}\right), \\
\hat{M}_{n} & =\frac{b z-\alpha_{1}+\alpha_{2}-\alpha_{3}}{(a-1) b} \frac{d}{d z}+1,
\end{aligned}
$$

where $U_{n}$ are some constants to be defined below, and $w_{n}$ is a set of special functions, their properties will be discussed below.

For what follows, it should be noted that the differential operator $\hat{H}_{n}$ satisfies the identity: 


$$
\begin{aligned}
\hat{H}_{n} \hat{M}_{n} \equiv & \hat{B}_{n} \hat{R}_{n}, \quad \hat{R}_{n}=\frac{d^{2}}{d z^{2}}+\left(\frac{2 \alpha_{1}}{z+1}+\frac{2 \alpha_{2}}{z-1}\right) \frac{d}{d z}+\frac{(a-1) b}{z^{2}-1}, \\
\hat{B}_{n}= & \frac{b z-\left(\alpha_{1}-\alpha_{2}+\alpha_{3}\right)}{(a-1) b} \frac{d}{d z}+\frac{2\left(\alpha_{1}+\alpha_{2}+1\right)-b}{a-1} \\
& +\frac{1}{(a-1) b}\left[\frac{\alpha_{1}-\alpha_{2}+\alpha_{3}}{z}+\frac{b-\left(\alpha_{1}-\alpha_{2}+\alpha_{3}\right)}{z-1}-\frac{b+\left(\alpha_{1}-\alpha_{2}+\alpha_{3}\right)}{z+1}\right],
\end{aligned}
$$

where imaginary parameters $a$ and $b$ have the form:

$$
a=\alpha_{1}+\alpha_{2}-\sqrt{2\left(\alpha_{1}^{2}+\alpha_{2}^{2}\right)-\alpha_{3}^{2}}, \quad b=\alpha_{1}+\alpha_{2}+\sqrt{2\left(\alpha_{1}^{2}+\alpha_{2}^{2}\right)-\alpha_{3}^{2}} .
$$

Then, we chose the functions $w_{n}$ satisfying the equation:

$$
\hat{R} w_{n}(\xi)=0, \quad \hat{R}=\frac{d^{2}}{d \xi^{2}}+\left(\frac{2 \alpha_{1}}{\xi}+\frac{2 \alpha_{2}}{\xi-1}\right) \frac{d}{d \xi}+\frac{(a-1) b}{\xi(\xi-1)}, \quad \xi=\frac{z+1}{2}
$$

which admits solutions in terms of hypergeometric functions. Taking into account Eq. (15), it is a trivial matter to show that functions (12) obey the initial equation (11).

Let us find the general solution of the hypergeometric equation (15). To this end we use two pairs of linearly independent solutions that we denote as $w_{n, i}(\xi)$; here additional indices $i=1, \ldots, 4$, are introduced to distinguish between solutions with the same quantum numbers $n$. Solutions $w_{n, 1}(\xi)$ and $w_{n, 2}(\xi)$ are

$$
\begin{aligned}
w_{n, 1}(\xi)= & \xi^{a-2 \alpha_{1}-1}(1-\xi)^{2 \alpha_{1}-a-b+1} \\
& \times F\left(2 \alpha_{1}-a+1,2-a ; 2 \alpha_{1}-a-b+2 ;\right. \\
& \left.2-\alpha_{1} ; 1-\xi^{-1}\right), \\
w_{n, 2}(\xi)= & \xi^{1-a} F\left(a-1, a-2 \alpha_{1} ; a+b-2 \alpha_{1} ; 1-\xi^{-1}\right) .
\end{aligned}
$$

Functions $F(\alpha, \beta ; \gamma ; \xi)^{2}$ are Gaussian hypergeometric functions [39]. Solutions (16) are well-defined in a vicinity of the singular point $\xi=1$ (which corresponds to $t \rightarrow-\infty$ ). Solutions $w_{n, 3}(\xi)$ and $w_{n, 4}(\xi)$ are

$$
\begin{aligned}
& w_{n, 3}(\xi)=(-\xi)^{-b} F\left(b, b-2 \alpha_{1}+1 ; b-a+2 ; \xi^{-1}\right), \\
& w_{n, 4}(\xi)=(-\xi)^{1-a} F\left(a-1, a-2 \alpha_{1} ; a-b ; \xi^{-1}\right) .
\end{aligned}
$$

They are well defined in a vicinity of the singular point $\xi=\infty$ (which corresponds to $t \rightarrow+\infty$ ). Using functions (16) and (17) we construct four complete sets $\varphi_{n, i}(t), i=1$, 2, 3, 4, of the corresponding solutions of Eq. (9).

Now one can move on to building the so-called in- and out-solutions $\psi_{n}(X)$ of the Dirac equation. These solutions have special asymptotics as $t \rightarrow \pm \infty$ and correspond to initial or final particles and antiparticles. The functions $\varphi(t)$ that correspond to spinors $\psi(t)$, that are in-solutions, are denoted as ${ }_{\zeta} \varphi_{n}(t)$, while functions $\varphi(t)$ that correspond to spinors $\psi(t)$, that are out-solutions, are denoted as ${ }^{\zeta} \varphi_{n}(t)$. Both sets are classified by a quantum number $\zeta= \pm$ which labels particles $(\zeta=+)$ and antiparticles $(\zeta=-)$. The electric field (4) vanishes at $|t| \rightarrow \infty$, but its vector potentials are different at $t \rightarrow-\infty$ and $t \rightarrow+\infty$, see Eq. (4). The above mentioned solutions ${ }_{\zeta} \varphi_{n}(t)$ and ${ }^{\zeta} \varphi_{n}(t)$ have the following asymptotic behavior,

$$
\begin{array}{rlrl}
{ }^{\zeta} \varphi_{n}(t) & ={ }^{\zeta} \mathcal{N} \exp \left(-i^{\zeta} \varepsilon_{n} t\right), & { }^{\zeta} \varepsilon_{n}=\zeta \omega_{1}, & t \rightarrow+\infty, \\
{ }_{\zeta} \varphi_{n}(t) & ={ }_{\zeta} \mathcal{N} \exp \left(-i_{\zeta} \varepsilon_{n} t\right), & \zeta^{\varepsilon_{n}}=\zeta \omega_{2}, \quad t \rightarrow-\infty, \\
\omega_{1} & =\sqrt{p_{x}^{2}+\pi_{\perp}^{2}}, \quad \omega_{2}=\sqrt{\left(p_{x}+e E_{0} \sigma\right)^{2}+\pi_{\perp}^{2}},
\end{array}
$$

where ${ }_{\zeta} \mathcal{N}$ and ${ }^{\zeta} \mathcal{N}$ are normalization constants.

Solutions (10) with the asymptotic conditions (18) have the following form:

$$
\begin{aligned}
& { }_{+} \varphi_{n}(t)={ }_{+} \mathcal{N} U_{n, 1}(1+z)^{\alpha_{1}}(1-z)^{\alpha_{2}} \hat{M}_{n} w_{n, 1}\left(\frac{z+1}{2}\right), \\
& { }_{-} \varphi_{n}(t)={ }_{-} \mathcal{N} U_{n, 2}(1+z)^{\alpha_{1}}(1-z)^{\alpha_{2}} \hat{M}_{n} w_{n, 2}\left(\frac{z+1}{2}\right), \\
& { }_{+} \varphi_{n}(t)={ }^{+} \mathcal{N} U_{n, 3}(1+z)^{\alpha_{1}}(1-z)^{\alpha_{2}} \hat{M}_{n} w_{n, 3}\left(\frac{z+1}{2}\right), \\
& { }^{-} \varphi_{n}(t)={ }^{-} \mathcal{N} U_{n, 4}(1+z)^{\alpha_{1}}(1-z)^{\alpha_{2}} \hat{M}_{n} w_{n, 4}\left(\frac{z+1}{2}\right),
\end{aligned}
$$

\footnotetext{
${ }^{2}$ These functions are also often denoted as ${ }_{2} F_{1}(\alpha, \beta ; \gamma ; \xi)$.
}

where the constants $U_{n, i}, i=1,2,3,4$, are 


$$
\begin{aligned}
U_{n, 1} & =\frac{2^{1-\alpha_{1}-3 \alpha_{2}} e^{i \pi \alpha_{2}}(a-1) b}{\left(2 \alpha_{2}-1\right)\left(b-\alpha_{1}+\alpha_{2}-\alpha_{3}\right)}, \\
U_{n, 2} & =\frac{2^{\alpha_{2}-\alpha_{1}+2} e^{-i \pi \alpha_{2}} \alpha_{2}}{a-\alpha_{1}+\alpha_{2}+\alpha_{3}}, \\
U_{n, 3} & =\frac{2^{-b} e^{-i \pi\left(\alpha_{2}-b\right)} a-1}{a-b-1}, \\
U_{n, 4} & =\frac{2^{1-a} e^{-i \pi\left(\alpha_{2}-a\right)} b(a-b)}{a\left(b-\alpha_{1}+\alpha_{2}-\alpha_{3}\right)-b\left(a+\alpha_{1}-\alpha_{2}-\alpha_{3}\right)} .
\end{aligned}
$$

The linear independence of solutions ${ }^{\zeta} \varphi_{n}(t)$ and ${ }_{\zeta} \varphi_{n}(t)$ (19) with different $\zeta$ can be proved as follows: using well-known relation (A3), given in Appendix, one sees that Wronskians of the functions $\varphi$ are proportional to Wronskians of the functions $w$, namely,

$$
\begin{aligned}
W\left({ }_{-} \varphi,{ }_{+} \varphi\right)= & W\left(_{+} \varphi,{ }_{-} \varphi\right)=\Omega_{n}(z) W\left(w_{n, 1}, w_{n, 2}\right), \\
W\left({ }^{-} \varphi,{ }^{+} \varphi\right)= & W\left({ }^{+} \varphi,{ }^{-} \varphi\right)=\Omega_{n}(z) W\left(w_{n, 3}, w_{n, 4}\right), \\
\Omega_{n}(z)= & 2^{2\left(\alpha_{1}+\alpha_{2}\right)}(1+z)^{2 \alpha_{1}}(1-z)^{2 \alpha_{2}} \\
& \times \frac{(a-b) \alpha_{3}+2\left(\alpha_{1}^{2}-\alpha_{2}^{2}\right)}{4(a-1) b} .
\end{aligned}
$$

In the case under consideration we have $W\left(w_{n, 1}, w_{n, 2}\right)=$ $W\left(w_{n, 3}, w_{n, 4}\right)=0$, which implies $W\left({ }_{\zeta} \varphi,{ }_{-\zeta} \varphi\right)=W\left({ }^{\zeta} \varphi\right.$, $\left.{ }^{-\zeta} \varphi\right)=0$ and, thus, proves the linear independence of the corresponding functions $\varphi$.

Since a second-order ordinary linear equation has two linearly independent solutions, all solutions with the same quantum numbers $n$ are found and they form complete sets.
We denote by $\left\{{ }_{\zeta} \psi_{n}(X)\right\}$ and by $\left\{\xi_{n} \psi_{n}(X)\right\}$ in- and outsolutions of Dirac equation (5) which are constructed via ${ }_{\zeta} \varphi_{n}(t)$ and ${ }^{\zeta} \varphi_{n}(t)$ correspondingly by the help of Eqs. (6) and (7).

Using the equal-time inner product (which is timeindependent for bispinors under consideration)

$$
(\psi, \psi)=\int d \mathbf{r} \psi^{\dagger}(X) \psi(X), \quad d \mathbf{r}=d x^{1} d x^{2} \ldots d x^{D}
$$

of Dirac bispinors, we easily calculate the normalization constants ${ }_{\zeta} \mathcal{N}$ and $\zeta \mathcal{N}$, using explicit forms of their asymptotics,

$$
\begin{array}{ll}
{ }^{\zeta} \mathcal{N}={ }^{\zeta} C Y, & { }^{\zeta} C=\left[2 \omega_{1}\left(\omega_{1}-\chi \zeta p_{x}\right)\right]^{-1 / 2}, \quad Y=V_{(d-1)}^{-1 / 2}, \\
{ }_{\zeta} \mathcal{N}={ }_{\zeta} C Y, & { }_{\zeta} C=\left\{2 \omega_{2}\left[\omega_{2}-\chi \zeta\left(p_{x}+e E_{0} \sigma\right)\right]\right\}^{-1 / 2} .
\end{array}
$$

In doing this, we use the standard volume regularization in which the r-integration in Eq. (22) is over a large spatial box of the volume $V_{(d-1)}=L_{1} \times \cdots \times L_{D}$ in $D$ dimensional Euclidean space, in this case, periodic boundary conditions are assumed for the Dirac bispinors. At the same time, one can see that the in- and out-solutions with different quantum numbers $n$ are orthogonal.

One can also see that in-solutions with quantum numbers $n$ are expressed via out-solutions with the same quantum numbers $n$. Thus,

$$
\zeta \psi_{n}(t)=\sum_{\zeta^{\prime}} g_{n}\left(\left.{ }_{\zeta^{\prime}}\right|^{\zeta}\right)_{\zeta^{\prime}} \psi_{n}(t)
$$

Coefficients $g\left(\left.{ }_{\zeta^{\prime}}\right|^{\zeta}\right)$ can be found with the help of the inner product (22),

$$
\left({ }_{\zeta} \psi_{n}, \zeta^{\prime} \psi_{n^{\prime}}\right)=g_{n}\left(\left.\zeta^{\prime}\right|^{\zeta}\right) \delta_{n n^{\prime}}, \quad g_{n}\left(\left.{ }_{\zeta^{\prime}}\right|^{\zeta}\right)=g_{n}\left(\left.{ }^{\zeta}\right|_{\zeta^{\prime}}\right)^{*}, \quad \sum_{\zeta^{\prime}} g_{n}\left(\left.{ }^{\zeta}\right|_{\zeta^{\prime}}\right) g_{n}\left(\left.\zeta_{\zeta^{\prime}}\right|^{\zeta^{\prime \prime}}\right)=\delta_{\zeta \zeta^{\prime \prime}} .
$$

Equations (23) and (24) imply the following decomposition of the corresponding scalar functions:

$$
{ }_{\zeta} \varphi_{n}(t)=g_{n}\left(\left.{ }^{+}\right|_{\zeta}\right)^{+} \varphi_{n}(t)+g_{n}\left(\left.{ }^{-}\right|_{\zeta}\right)^{-} \varphi_{n}(t) .
$$

Using the Kummer relations (A9) and (A10) for the hypergeometric equation [39] and decompositions (25), we find the coefficients $g_{n}\left(\zeta_{\zeta^{\prime}}\right)$ to be

$$
\begin{aligned}
& g_{n}\left(\left.{ }^{+}\right|_{+}\right)=\frac{{ }_{+} \mathcal{N}}{{ }^{+} \mathcal{N}} \frac{2^{b-\alpha_{1}-3 \alpha_{2}+1} \sin (\pi b) \Gamma(a-b) \Gamma(b+1)}{\left(b-\alpha_{1}+\alpha_{2}-\alpha_{3}\right) \sin \left(2 \pi \alpha_{2}\right) \Gamma\left(2 \alpha_{1}-b\right) \Gamma\left(2 \alpha_{2}\right)}, \\
& g_{n}\left(\left.{ }^{-}\right|_{+}\right)=-{ }_{+} \mathcal{N} \mathcal{N} \frac{2^{a-\alpha_{1}-3 \alpha_{2}}\left(a-\alpha_{1}+\alpha_{2}+\alpha_{3}\right) \sin (\pi a) \Gamma(b-a) \Gamma(a)}{\sin \left(2 \pi \alpha_{2}\right) \Gamma\left(b-2 \alpha_{2}+1\right) \Gamma\left(2 \alpha_{2}\right)}, \\
& g_{n}\left(\left.{ }^{+}\right|_{-}\right)=-\frac{-\mathcal{N}}{{ }^{+} \mathcal{N}} \frac{2^{b-\alpha_{1}+\alpha_{2}+1} \pi \Gamma(a-b)}{\left(a-\alpha_{1}+\alpha_{2}+\alpha_{3}\right) \sin \left(2 \pi \alpha_{2}\right) \Gamma(a) \Gamma\left(-2 \alpha_{2}\right) \Gamma\left(a-2 \alpha_{1}\right)}, \\
& g_{n}\left(\left.{ }^{-}\right|_{-}\right)=-\frac{-\mathcal{N}}{-\mathcal{N}} \frac{2^{a-\alpha_{1}+\alpha_{2}} \pi\left(b-\alpha_{1}+\alpha_{2}-\alpha_{3}\right) \Gamma(b-a)}{\sin \left(2 \pi \alpha_{2}\right) \Gamma(b+1) \Gamma\left(-2 \alpha_{2}\right) \Gamma\left(1-a+2 \alpha_{2}\right)},
\end{aligned}
$$

where $\Gamma(x)$ is the gamma-function. 


\section{VACUUM INSTABILITY CHARACTERISTICS}

Here, using exact solutions that were found above, we already can calculate characteristics of the vacuum instability in the electric field (4), namely the vacuum-tovacuum transition probability $P_{\mathrm{v}}$, differential $N_{n}$ and total $N$ mean numbers of created pairs. As it follows from the general formulation of strong-field $Q E D$ with $t$-electric potential steps, all these characteristics are expressed via coefficients (26),

$P_{\mathrm{v}}=\exp \left[\sum_{n} \ln \left(1-N_{n}\right)\right], \quad N_{n}=\left|g_{n}\left(\left.{ }_{-}\right|^{+}\right)\right|^{2}, \quad N=\sum_{n} N_{n}$.

First, using Eqs. (26), we find the differential numbers $N_{n}$. They are

$N_{n}=\frac{\sinh 2 \sigma \pi\left(\omega_{0}+\omega_{1}-\omega_{2} / 2\right) \sinh 2 \sigma \pi\left(\omega_{0}-\omega_{1}+\omega_{2} / 2\right)}{\sinh 4 \sigma \pi \omega_{1} \sinh 2 \sigma \pi \omega_{2}}$,

$\omega_{0}=\frac{1}{2} \sqrt{\left(p_{x}-e E_{0} \sigma\right)^{2}+\pi_{\perp}^{2}}$,

$\omega_{1}=\sqrt{p_{x}^{2}+\pi_{\perp}^{2}}, \quad \omega_{2}=\sqrt{\left(p_{x}+e E_{0} \sigma\right)^{2}+\pi_{\perp}^{2}}$.

To further analysis it is convenient to use the gauge invariant longitudinal kinetic momentum, $P_{x}(t)=p_{x}+$ $e A_{x}(t)$ and the increment $\Delta W$ of the longitudinal kinetic momentum,

$$
\Delta W=P_{x}(t \rightarrow-\infty)-P_{x}(t \rightarrow+\infty) .
$$

In the case under consideration we have $\Delta W=e E_{0} \sigma$.

Let us analyze the dependence of the calculated quantities on the parameter $\sigma$, which determines the shape of the analytic asymmetric electric field. First, we consider small values of the parameter $\sigma$,

$$
\sigma \ll\left(e E_{0}\right)^{-1} \sqrt{p_{x}^{2}+\pi_{\perp}^{2}} .
$$

In this case, the electric field (4) and its potential change rapidly, and the electric field is a short pulse corresponding to a small increment $\Delta W$. As it follows from Eq. (28), in this case, the differential mean numbers $N_{n}$ are also small enough for any $p_{x}$ and $\pi_{\perp}$,

$$
N_{n}=\frac{\left(e E_{0} \sigma\right)^{2} \pi_{\perp}^{2}}{4\left(p_{x}^{2}+\pi_{\perp}^{2}\right)^{2}}\left[1+O\left(\frac{e E_{0} \sigma}{p_{x}^{2}+\pi_{\perp}^{2}}\right)\right] .
$$

It is the case of a weak external field such a result can be derived in the framework of perturbation theory with respect to the external field. At small longitudinal momenta, $p_{x}^{2} \ll \pi_{\perp}^{2}$, expression (31) is reduced to the one

$$
N_{n} \approx \frac{(\Delta W)^{2}}{4 \pi_{\perp}^{2}},
$$

which coincides with the result obtained, for example, for a weak pulse of $T$-constant electric field with the height $\Delta W=e E T$ of a corresponding step in the same range of longitudinal momenta (see [36]). Since the form of $T$-const field is quite different from the one of the field (4), we conclude that in the case of a small $\Delta W$ the leading term of the distribution $N_{n}$ is given by Eq. (32) that depends only on $\Delta W$ and does not depend on the field configuration.

As it follows from a semiclassical consideration, most particles produced at a time instant $t$ have zero longitudinal kinetic momenta and then are accelerated by a field. Thus, we expect to find maximum of the distribution $N_{n}$ when longitudinal kinetic momenta at time $t_{\max }$,

$$
P_{x}\left(t_{\max }\right)=p_{x}^{\prime}, \quad p_{x}^{\prime}=p_{x}+e E_{0} \sigma / \sqrt{3},
$$

is zero. Because of that in what follows we use value $p_{x}^{\prime}$, which is best suited for analysis of $N_{n}$.

Parameters $\omega$ (28), being written in terms $p_{x}^{\prime}$, have the form:

$$
\begin{aligned}
& \omega_{0}=\frac{1}{2} \sqrt{\left[p_{x}^{\prime}-e E_{0} \sigma(1+1 / \sqrt{3})\right]^{2}+\pi_{\perp}^{2}}, \\
& \omega_{1}=\sqrt{\left(p_{x}^{\prime}-e E_{0} \sigma / \sqrt{3}\right)^{2}+\pi_{\perp}^{2}}, \\
& \omega_{2}=\sqrt{\left[p_{x}^{\prime}+e E_{0} \sigma(1-1 / \sqrt{3})\right]^{2}+\pi_{\perp}^{2}} .
\end{aligned}
$$

Let us consider the electric field for which $\sigma \rightarrow 0$ and $E_{0}^{-1} \rightarrow 0$, such that the increment $\Delta W=e E_{0} \sigma$ is a finite quantity. At the same time, we assume that for sufficiently small $\sigma$ parameters $\omega_{0}, \omega_{1}$ and $\omega_{2}$ satisfy the following inequalities:

$$
\Delta W \sigma \ll 1, \quad \max \left\{\sigma \omega_{0}, \sigma \omega_{1}, \sigma \omega_{2}\right\} \ll 1 .
$$

In this case, one can approximate the mean numbers $N_{n}$ as:

$$
N_{n} \approx \frac{\omega_{0}^{2}-\left(\omega_{1}-\omega_{2} / 2\right)^{2}}{2 \omega_{2} \omega_{1}} .
$$

If the increment $\Delta W$ is large enough, $\Delta W \gg \pi_{\perp}, p_{x}^{\prime}$, then one can represent Eq. (36) as follows:

$$
N_{n}=1+O\left(\max \left[\frac{p_{x}^{\prime}}{\Delta W}, \frac{\pi_{\perp}^{2}}{(\Delta W)^{2}}\right]\right) .
$$

We see that in this case the differential mean numbers $N_{n}$ reach the maximum possible value for fermions $N_{n} \approx 1$, in a wide range of the momenta $p_{x}^{\prime}$ and $\pi_{\perp}$. The width of each of these ranges is only one order less that $\Delta W$. Note that this is a characteristic feature of short strong pulses with 
large potential steps, which can be observed in all exactly solvable cases, see review [18].

In what follows we consider the case of big $\sigma$,

$$
\sigma \gg\left(e E_{0}\right)^{-1 / 2} \max \left\{1, m^{2} / e E_{0}\right\},
$$

which corresponds to a slowly varying electric field,

$2 \sigma \pi\left[\omega_{0} \pm\left(\omega_{1}-\omega_{2} / 2\right)\right] \gg 1, \quad 4 \sigma \pi \omega_{1} \gg 1, \quad 4 \sigma \pi \omega_{2} \gg 1$.

In this case the differential mean numbers (28) can be approximately presented as:

$$
N_{n} \approx \exp [-\pi \tau], \quad \tau=2 \sigma\left(2 \omega_{1}+\omega_{2}-2 \omega_{0}\right) .
$$

One can check that the mean numbers $N_{n}$ (28) are negligibly small,

$$
N_{n} \ll e^{-\pi m^{2} / e E_{\max }},
$$

in ranges where initial and final longitudinal kinetic momenta $P_{x}(-\infty)$ and $P_{x}(+\infty)$ are not large,

$$
\begin{aligned}
\left|p_{x}^{\prime}-e E_{0} \sigma / \sqrt{3}\right| & <\sqrt{e E_{0}} K, \\
\left|p_{x}^{\prime}+e E_{0} \sigma(1-1 / \sqrt{3})\right| & <\sqrt{e E_{0}} K,
\end{aligned}
$$

or are too large,

$$
\begin{aligned}
& \sqrt{e E_{0}} K<p_{x}^{\prime}-e E_{0} \sigma / \sqrt{3} \\
& \sqrt{e E_{0}} K<-\left[p_{x}^{\prime}+e E_{0} \sigma(1-1 / \sqrt{3})\right] .
\end{aligned}
$$

Thus, one can conclude that main contributions to the mean numbers $N_{n}$ originate from the range of momentum $p_{x}^{\prime}$ determined by the double inequality:

$\left[\sqrt{e E_{0}} K-e E_{0} \sigma(1-1 / \sqrt{3})\right]<p_{x}^{\prime}<e E_{0} \sigma / \sqrt{3}-\sqrt{e E_{0}} K$.

Let us turn to Eq. (40). The function $\tau$ has a minimum at $p_{x}^{\prime}=0$, which corresponds to the mean number $N_{n}$ of created particles by a constant uniform field with $E(t)=E_{\max }$, then $\tau$ grows monotonically as both $\left|p_{x}^{\prime}\right|$ and $\pi_{\perp}$ grow. One can show that the mean number $N_{n}$ is exponentially small in the range of large transversal momenta, $\pi_{\perp} \gtrsim \sqrt{e E_{0}} K$. Therefore the following range of $\pi_{\perp}$ is of interest:

$$
\pi_{\perp} \ll \sqrt{e E_{0}} K .
$$

Conditions (44) and (45) determine a range $\Omega$ of momenta, beyond which the distribution $N_{n}$ is negligible. In this range, the following approximation of the parameter $\tau$ holds true:

$$
\begin{aligned}
N_{n} & \approx N_{n}^{\text {as }}=\exp (-\pi \tau), \\
\tau & \approx \frac{18 \sigma \pi_{\perp}^{2}\left(e E_{0} \sigma\right)^{2}}{2 \sqrt{3}\left(e E_{0} \sigma\right)^{3}-9 \sqrt{3} e E_{0} \sigma p_{x}^{\prime 2}+9 p_{x}^{\prime 3}} .
\end{aligned}
$$

One can see that in two limiting cases:

$$
\begin{aligned}
e E_{0} \sigma / \sqrt{3}-p_{x}^{\prime} & \rightarrow \sqrt{e E_{0}} K, \\
p_{x}^{\prime}+e E_{0} \sigma(1-1 / \sqrt{3}) & \rightarrow \sqrt{e E_{0}} K,
\end{aligned}
$$

either $\omega_{1}$ or $\omega_{2}$ reach their maxima and the function $\tau$ reaches its maximal values $\tau_{\max }^{+}$or $\tau_{\max }^{-}$respectively. One can see that $\tau_{\max }^{ \pm} \rightarrow \infty$ as $\sqrt{e E_{0}} \sigma \rightarrow \infty$.

In a wide range of transversal and longitudinal momenta, $\pi_{\perp} \ll e E_{0} \sigma, p_{x}^{\prime} \ll e E_{0} \sigma$, the differential mean numbers $N_{n}$ do not depend on the parameter $\sigma$ and coincide with ones in the constant electric field $E_{\max }$, which are

$N_{n} \approx N_{n}^{0}=e^{-\pi \tau_{0}}, \quad \tau_{0}=\left.\tau\right|_{p_{x}^{\prime}=0}=\lambda=\frac{\pi_{\perp}^{2}}{e E_{\max }}$,

see Refs. $[2,40]$.

The total number of pairs created from the vacuum ( $N=\sum_{n} N_{n}$ ) by a uniform electric field, is proportional to the space volume $V_{(d-1)}$ as $N=V_{(d-1)} \rho$. One can see that the number density $\rho$ has the form:

$$
\rho=\frac{1}{(2 \pi)^{d-1}} \sum_{s} \int N_{n} d \mathbf{p} .
$$

In deriving Eq. (49) the sum over all momenta $p$ was transformed into an integral. Then the integral in the right hand side of Eq. (49) can be approximated by an integral over a subrange $\Omega$ (given by Eqs. (44) and (45) that represents a dominant contribution with respect to the total increment to the number density of created particles,

$$
\Omega: \rho^{\Omega}=\frac{1}{(2 \pi)^{d-1}} \sum_{s} \int_{\mathbf{p} \in \Omega} N_{n} d \mathbf{p} .
$$

This quantity can be calculated using Eq. (50) with differential numbers $N_{n}$ approximated by Eq. (46). In this case, the leading term $\rho^{\Omega}$ is formed over the range given by Eqs. (44) and (45). In this approximation, the mean numbers $N_{n}$ do not depend on the spin polarization parameters $s$. Thus, the summation over $s$ produces the factor $J_{(d)}=2^{[d / 2]-1}$ (the number of spin degrees of freedom), such that:

$$
\rho^{\Omega}=\frac{J_{(d)}}{(2 \pi)^{d-1}} \int_{\mathbf{p} \in \Omega} N_{n} d \mathbf{p} .
$$

Taking into account Eq. (46), we approximate integral (51) as: 


$$
\begin{aligned}
\rho^{\Omega} & \approx \frac{J_{(d)}}{(2 \pi)^{d-1}} \int\left(I_{p_{\perp}}^{+}+I_{p_{\perp}}^{-}\right) d \mathbf{p}_{\perp}, \\
I_{p_{\perp}}^{+} & =\int_{0}^{e E_{0} \sigma / \sqrt{3}-\sqrt{e E_{0}} K} e^{-\pi \tau} d p_{x}^{\prime}, \\
I_{p_{\perp}}^{-} & =\int_{-\left[e E_{0} \sigma(1-1 / \sqrt{3})-\sqrt{e E_{0}} K\right]}^{0} e^{-\pi \tau} d p_{x}^{\prime} .
\end{aligned}
$$

To calculate $I_{p_{\perp}}^{+}$and $I_{p_{\perp}}^{-}$, it is convenient to represent $\tau$ as follows $\tau=\lambda(q+1)$ and to pass from the integration over $p_{x}^{\prime}$ to the integration over a parameter $q$ (the transition to such a variable provides exponential decrease of the integrand with increasing $q$, and the expansion of the preexponential factor in powers of $q$ has the form of an asymptotic series). To this end we have to find $p_{x}^{\prime}$ as a function of $q$ using Eq. (46). Such a function can be found from the cubic equation

$$
r^{3}-\sqrt{3} r^{2}+\frac{2 q}{3 \sqrt{3}(q+1)}=0, \quad r=\frac{p_{x}^{\prime}}{e E_{0} \sigma}
$$

Note that when $p_{x}^{\prime} \rightarrow e E_{0} \sigma / \sqrt{3}-\sqrt{e E_{0}} K$ and $p_{x}^{\prime} \rightarrow$ $-\left[e E_{0} \sigma(1-1 / \sqrt{3})-\sqrt{e E_{0}} K\right]$, the parameter $\tau$ reaches the limiting values $\tau_{\max }^{ \pm}=\lambda\left(q_{\max }^{ \pm}+1\right)$ respectively. However, since contributions of the factor $\exp (-\pi \tau)$ to integrals (52) outside of range (44) are exponentially small, one can extend limits of the integration over $q$ to $\pm \infty$. Equation (53) has three real solutions:

$$
\begin{aligned}
& r_{1}=\frac{2}{\sqrt{3}} \cos \frac{\alpha(q)}{3}+1 / \sqrt{3}, \quad \alpha(q)=\arccos \left[(q+1)^{-1}\right] \\
& r_{2}=-\frac{2}{\sqrt{3}} \cos \left[\frac{\alpha(q)}{3}+\frac{\pi}{3}\right]+1 / \sqrt{3} \\
& r_{3}=-\frac{2}{\sqrt{3}} \cos \left[\frac{\alpha(q)}{3}-\frac{\pi}{3}\right]+1 / \sqrt{3}
\end{aligned}
$$

see, e.g., [41].

Since $0<q<+\infty$, the following inequality holds true $0 \leq \alpha(q) \leq \pi / 2$, which implies:

$$
\begin{aligned}
0 & \leq \frac{\alpha(q)}{3} \leq \frac{\pi}{6}, \quad \frac{\pi}{3} \leq\left[\frac{\alpha(q)}{3}+\frac{\pi}{3}\right] \leq \frac{\pi}{2}, \\
-\frac{\pi}{3} & \leq\left[\frac{\alpha(q)}{3}-\frac{\pi}{3}\right] \leq-\frac{\pi}{6} .
\end{aligned}
$$

Then

$$
1 \leq r_{1} \leq \sqrt{3}, \quad 0 \leq r_{2} \leq \frac{1}{\sqrt{3}}, \quad\left(\frac{1}{\sqrt{3}}-1\right) \leq r_{3} \leq 0
$$

such that solutions $r_{2}$ and $r_{3}$ represent the parameter $p_{x}^{\prime}$ in the subranges $p_{x}^{\prime} \in\left(-e E_{0} \sigma(1-1 / \sqrt{3}), 0\right)$ and $p_{x}^{\prime} \in\left(0, e E_{0} \sigma / \sqrt{3}\right)$, respectively.

Thus, the integrals $I_{p_{\perp}}^{+}$and $I_{p_{\perp}}^{-}$take the forms:

$$
I_{p_{\perp}}^{ \pm}= \pm \frac{2 \Delta W}{3 \sqrt{3}} \int_{0}^{+\infty} d q \frac{(q+1)^{-2}}{\sqrt{1-(q+1)^{-2}}} \sin \left[\frac{\alpha(q)}{3} \pm \frac{\pi}{3}\right] \exp [-\pi \lambda(q+1)]
$$

where $\Delta W=e E_{0} \sigma$, and their sum can be represented as:

$$
I_{p_{\perp}}^{-}+I_{p_{\perp}}^{+}=\frac{2}{3} \Delta W \int_{0}^{+\infty} d q \frac{(1+q)^{-2}}{\sqrt{1-(1+q)^{-2}}} \cos \frac{\alpha(q)}{3} \exp [-\pi \lambda(q+1)] .
$$

Substituting (58) into (52) and integrating over $d p_{\perp}^{(d-2)}$, we get:

$$
\begin{aligned}
& N \approx V_{(d-1)} \rho^{\Omega}, \quad \rho^{\Omega}=\beta \frac{\Delta W}{e E_{\max }} k, \quad \beta=\frac{J_{(d)}\left[e E_{\max }\right]^{d / 2}}{(2 \pi)^{d-1}} \exp \left[-\frac{\pi m^{2}}{e E_{\max }}\right], \\
& k=\frac{2}{3} \int_{0}^{+\infty} d q\left(q^{2}+2 q\right)^{-1 / 2}(q+1)^{-d / 2} \cos \frac{\alpha(q)}{3} \exp \left[-\frac{\pi m^{2}}{e E_{\max }} q\right] .
\end{aligned}
$$

The corresponding probability $P_{\mathrm{v}}$ of the vacuum to remain a vacuum reads:

$P_{\mathrm{v}}=\exp [-\mu N], \quad \mu=\sum_{l=0}^{\infty}(l+1)^{-d / 2} \exp \left(-l \frac{\pi m^{2}}{e E_{\max }}\right)$
In this approximation, it is possible to find explicitly additional characteristics of the vacuum instability, namely, leading terms in representations of vacuum means of the current density vector $\left\langle j^{\mu}(t)\right\rangle$ and of the energy-momentum tensor (EMT) $\left\langle T_{\mu \nu}(t)\right\rangle$, supposing that field (4) weakens at macroscopically large time $t=t_{\text {out }} \gg t_{\max }$; see, e.g., Ref. [34] for a field representation of these quantities. 
At this time instant, the solutions ${ }^{5} \psi_{n}(X)$ are reduced to free particle plane waves in agreement with Eq. (18). The renormalized vacuum polarization contributions to the quantities $\left\langle j^{\mu}(t)\right\rangle$ and $\left\langle T_{\mu \nu}(t)\right\rangle$ vanish in the absence of the external field. Therefore, for $t \geq t_{\text {out }}$, these vacuum means are constant and represent the mean current density and EMT of created particles. The latter quantities can be calculated via differential mean numbers $N_{n}$ given by Eq. (46). Dominant contributions to $\left\langle j^{\mu}\left(t_{\text {out }}\right)\right\rangle$ and $\left\langle T_{\mu \nu}\left(t_{\text {out }}\right)\right\rangle$ are formed in subranges $\Omega$, defined by Eqs. (44) and (45). Thus, the nonzero means are

$$
\left\langle j^{1}\left(t_{\text {out }}\right)\right\rangle \approx 2 e \rho^{\Omega},
$$

and

$$
\begin{aligned}
\left\langle T_{00}\left(t_{\text {out }}\right)\right\rangle \approx & \left\langle T_{11}\left(t_{\text {out }}\right)\right\rangle \approx \frac{J_{(d)}}{(2 \pi)^{d-1}} \frac{2(\Delta W)^{2}}{3 \sqrt{3}}\left(e E_{\max }\right)^{d / 2-1} \\
& \times \int_{0}^{+\infty} \frac{(1+q)^{-d / 2}}{\sqrt{q^{2}+2 q}} \cos \left[\frac{2 \alpha(q)}{3}\right] \exp \left[-\frac{\pi m^{2}}{e E_{\max }}(q+1)\right] d q, \\
\left\langle T_{l l}\left(t_{\text {out }}\right)\right\rangle \approx & \frac{J_{(d)}}{(2 \pi)^{d-1}} \frac{\left(e E_{\max }\right)^{d / 2}}{\sqrt{3}} \int_{0}^{+\infty} \frac{(1+q)^{-d / 2-1}}{\sqrt{q^{2}+2 q}} \\
& \times\left[\cos \frac{2 \alpha(q)}{3}-\frac{1}{2}\right]^{-1} \exp \left[-\frac{\pi m^{2}}{e E_{\max }}(q+1)\right] d q, \quad l=2, \ldots, D .
\end{aligned}
$$

We see that the mean number density $\rho^{\Omega}$ and the mean current density $\left\langle j^{1}\left(t_{\text {out }}\right)\right\rangle$ of created pairs are proportional to the increment $\Delta W$ of the kinetic momentum. This latter quantity defines the total number of states $\Delta W L / 2 \pi$ with the longitudinal momenta $p_{x}$, in which particles can be created (here $L$ is the length of the system along the axis $x$ ). The vacuum means $\left\langle T_{00}\left(t_{\text {out }}\right)\right\rangle \approx\left\langle T_{11}\left(t_{\text {out }}\right)\right\rangle$ are proportional to the square of the increment, while the vacuum means $\left\langle T_{l l}\left(t_{\text {out }}\right)\right\rangle$ do not depend on $\Delta W$ if the latter quantity is sufficiently large. Note that this is typical to any slowly varying field [34]. The latter property allows one to compare number densities of created pairs due to various slowly varying electric fields. Among all exactly solvable cases discussed above, a special place is occupied by the case of $T$-constant electric field which is constant within the large time interval $T$. The fields of other exactly solvable cases decrease to zero with distance from the corresponding maxima, so one may expect that in these cases the pair production efficiency will be lower. Thus, it is natural to compare the number density of created pairs by fields with equal $E_{\max }$ and increment $\Delta W$ with the case of $T$-constant field considered in detail in Refs. [36]. In the latter case we set $E_{\max }=E$ and $\Delta W=e E T$. The density of created pairs due to $T$-constant field reads:

$$
\rho_{T}^{\Omega}=\beta \frac{\Delta W}{e E_{\max }} .
$$

It is a linear function of the time duration $T$ and the quantity $\beta$, given by Eq. (59), is the pairs production rate. In the exactly solvable cases with Sauter-like electric field (3) and in the peak field configurations of the exponential electric field (see Refs. $[18,19]$ ) the latter quantities are (i) $\rho_{S}^{\Omega}=\rho_{T}^{\Omega} k_{S}$ for Sauter-like field,

(ii) $\rho_{\mathrm{p}}^{\Omega}=\rho_{T}^{\Omega} k_{\mathrm{p}}$ for exponential peak field,

(iii) $\rho_{\mathrm{sq}}^{\Omega}=\rho_{T}^{\Omega} k_{\mathrm{sq}}$ for inverse square peak field,

where

$$
\begin{aligned}
k_{S} & =\frac{1}{2} \int_{0}^{\infty} d q q^{-1 / 2}(q+1)^{-(d+1) / 2} \exp \left(-q \pi \frac{m^{2}}{e E_{\max }}\right) \\
k_{\mathrm{p}} & =\int_{0}^{\infty} \frac{d q}{(q+1)^{d / 2+1}} \exp \left(-q \pi \frac{m^{2}}{e E_{\max }}\right) \\
k_{\mathrm{sq}} & =\frac{1}{2} \int_{0}^{\infty} \frac{d q}{(q+1)^{d / 2}} \exp \left(-q \pi \frac{m^{2}}{e E_{\max }}\right) .
\end{aligned}
$$

Formulas (59) and (65) show how differences in the shapes of $t$-steps affect the integrands for factors $k$ 's. These factors for a not so strong electric field $\left(\mathrm{m}^{2} / e E_{\max }>1\right)$ can be approximated as:

$k \approx \frac{\sqrt{2}}{3} k_{S}, \quad k_{S} \approx \frac{\sqrt{e E_{\mathrm{max}}}}{m}, \quad k_{\mathrm{sq}} \approx \frac{1}{2} k_{\mathrm{p}}, \quad k_{\mathrm{p}} \approx \frac{e E_{\mathrm{max}}}{\pi m^{2}}$.

In this case, we see that the number density of created pairs by analytic asymmetric and Sauter-like fields comparable to each other but less the density $\rho_{T}^{\Omega}$ in $T$-constant field by the order factor $\sqrt{e E_{\max }} / \mathrm{m}$. The mean number densities $\rho_{\mathrm{p}}^{\Omega}$ and $\rho_{\mathrm{sq}}^{\Omega}$ are comparable to each other, but due to the factor $e E_{\max } / m^{2}$ are less than the density $\rho_{T}^{\Omega}$. The obtained estimates mean that for the case of not very strong electric 
fields, vacuum instability effects by the analytic asymmetric and Sauter-like fields decrease over time, deviating from their maximum values, much more slowly than by sharppeak fields.

In the case of a strong electric field $\left(\pi \mathrm{m}^{2} / e E_{\max } \ll 1\right)$ exponential factors in integrals (65) can be approximated by units. Then, in this approximation, the factors $k$ 's differ slightly and have the form:

$$
\begin{aligned}
k & \approx\left\{\begin{array}{l}
0.65 \text { if } d=4 \\
0.77 \text { if } d=3
\end{array}, \quad k_{S} \approx\left\{\begin{array}{l}
0.67 \text { if } d=4 \\
0.79 \text { if } d=3
\end{array},\right.\right. \\
k_{\mathrm{sq}} & \approx \frac{1}{d-1}, \quad k_{\mathrm{p}} \approx \frac{2}{d} .
\end{aligned}
$$

Thus, if all the above mentioned $t$-steps are strong enough, intensities of the corresponding pair productions are quite similar.

\section{SLOWLY VARYING FIELD APPROXIMATION}

A new semiclassical approximation approach that is not restricted by a smallness of differential mean numbers of created pairs was recently proposed for treating the vacuum instability in strong-field $Q E D$ with $t$-steps slowly varying with time [34]. This approach is closely related to the leading term approximation of derivative expansion in field-theoretic calculations [42-45] (see Ref. [46] for a review). In fact, it is an extension of a locally constant field approximation (LCFA) for calculating vacuum mean values of physical quantities. It maintains the nonperturbative character of $Q E D$ calculations even in the absence of the exact solutions. In this approximation one can see an universal character of the vacuum effects caused by a strong electric field, defining the slowly varying regime in general terms. In particular, one finds representations for the total density of created pairs and vacuum mean values of the current density and energy-momentum tensor as a functional of an external electric field. In this section we compare the results of such an approximation to ones elaborated from exact solutions presented above for sufficiently large $\sigma$.
We call $E(t)$ a slowly varying electric field on a time interval $\Delta t$ from $t$ to $t+\Delta t$ if the following condition holds true:

$$
\left|\frac{\overline{\dot{E}(t)} \Delta t}{\overline{E(t)}}\right| \ll 1
$$

where $\overline{E(t)}$ and $\overline{\dot{E}(t)}$ are mean values of $E(t)$ and $\dot{E}(t)$ on the time interval $\Delta t$, respectively, and $\Delta t$ is significantly larger than the time scale $\Delta t_{\mathrm{sc}}$,

$$
\begin{aligned}
\Delta t / \Delta t_{\mathrm{sc}} & \gg 1, \\
\Delta t_{\mathrm{sc}} & =[e \overline{E(t)}]^{-1 / 2} \max \left\{1, m^{2} / e \overline{E(t)}\right\} .
\end{aligned}
$$

Property (67) is inherent to the field (4) for sufficiently large $\sigma$ satisfying condition (38) and for $\Delta t$ satisfying both Eq. (68) and the condition

$$
\Delta t / \sigma \ll 1
$$

In this case one can approximate the mean value $\overline{E(t)}$ in the time interval $\Delta t$ as $\overline{E(t)} \approx E(t)$. For a given $p_{\perp}$ one can consider the time interval $\Delta t$ as a sufficiently large if

$\Delta t \sqrt{e E(t)} \gg \max \{1, \lambda(t)\}, \quad \lambda(t)=\pi_{\perp}^{2} / e E(t)$.

Since the field under consideration (as all the above mentioned fields) weakens as $t \rightarrow \pm \infty$ there always exist some time instants $t_{\text {in }}$ and $t_{\text {out }}$ such that for any $\Delta t$, which satisfies condition (69), the parameter $\lambda(t)$ achieves critical values $\lambda_{\text {out } / \text { in }}=\Delta t \sqrt{e E\left(t_{\text {out } / \text { in }}\right)}>1$ respectively. For big $\lambda(t)$, satisfying inequalities $\lambda(t)>\lambda_{\text {out }}$ or $\lambda(t)>\lambda_{\text {in }}$, condition (70) is not valid. Because of this the slowly varying field approximation is applicable only in the domain of a strong enough field when $t_{\text {in }}<t<t_{\text {out }}$. However, the violation of the vacuum stability by a small electric field, $E(t)<E\left(t_{\text {out } / \text { in }}\right)$, is negligibly small.

In the domain of a strong enough field the leading term in the density $\rho^{\Omega}$ in the slowly varying field approximation reads:

$$
\rho^{\Omega} \approx \frac{J_{(d)}}{(2 \pi)^{d-1}} \int_{t_{\text {in }}}^{t_{\text {out }}} e E(t) d t \int d \mathbf{p}_{\perp} N_{n}^{\text {univ }}, \quad N_{n}^{\text {univ }}=\exp \left[-\pi \frac{\pi_{\perp}^{2}}{e E(t)}\right]
$$

and the probability of the vacuum to remain a vacuum has the form:

$$
P_{v} \approx \exp \left\{-\frac{V_{(d-1)} J_{(d)}}{(2 \pi)^{d-1}} \sum_{l=0}^{\infty} \int_{t_{\text {in }}}^{t_{\text {out }}} d t(-1)^{(1-\kappa) l / 2} \frac{[e E(t)]^{d / 2}}{(l+1)^{d / 2}} \exp \left[-\pi \frac{(l+1) m^{2}}{e E(t)}\right]\right\},
$$

respectively, with the field $E(t)$ given by Eq. (4). 
Let us compare these results with the ones presented in Eq. (57). One can represent Eq. (71) in the form

$$
\begin{aligned}
\rho^{\Omega} & \approx \frac{J_{(d)}}{(2 \pi)^{d-1}} \int d \mathbf{p}_{\perp}\left(J_{p_{\perp}}^{+}+J_{p_{\perp}}^{-}\right), \\
J_{p_{\perp}}^{+} & =\int_{t_{\max }}^{\infty} d t[e E(t)] N_{n}^{\text {univ }}, \quad J_{p_{\perp}}^{-}=\int_{-\infty}^{t_{\max }} d t[e E(t)] N_{n}^{\text {univ }} .
\end{aligned}
$$

The electric field $E(t)$, given by Eq. (4) is related to the quantity $W(t)$ as

$$
W(t)=\frac{-e E_{0} \sigma}{\sqrt{1+\exp (t / \sigma)}},
$$

such that $e E(t) d t=d W(t)$. On the other hand, one can relate the functions $E(t)$ and $W(t)$ via a cubic equation,

$$
y^{3}-y-2 E(t) / E_{0}=0,
$$

where the notation $y=W(t) /\left(e E_{0} \sigma\right)$ is used. It is convenient to introduce a variable $q$ such that

$$
q=\left(3 \sqrt{3} E(t) / E_{0}\right)^{-1}-1 .
$$

We can express $W(t)$ as a function of the field $E(t)$ or as a function of the variable $q$ using solutions of equation (74). This equation has three real solutions,

$$
\begin{aligned}
& y_{1}=\frac{2}{\sqrt{3}} \cos \frac{\alpha(q)}{3}, \quad y_{2}=-\frac{2}{\sqrt{3}} \cos \left[\frac{\alpha(q)}{3}+\frac{\pi}{3}\right], \\
& y_{3}=-\frac{2}{\sqrt{3}} \cos \left[\frac{\alpha(q)}{3}-\frac{\pi}{3}\right], \quad \alpha(q)=\arccos \left[(q+1)^{-1}\right],
\end{aligned}
$$

see, e.g., [41]. Since $W(t)$ is negative, only the solutions $y_{2,3}$ are relevant. One can see that for solutions $y_{2,3}$ the differential $d W(t)$ takes the form:

$$
d W(t)=\frac{2 e E_{0} \sigma}{3 \sqrt{3}} \sin \left[\frac{\alpha(q)}{3} \pm \frac{\pi}{3}\right] \frac{(1+q)^{-1} d q}{\sqrt{q^{2}+2 q}} .
$$

Passing from the integration over $t$ to the integration over the parameter $q$ in Eq. (73), we find:

$$
J_{p_{\perp}}^{ \pm}=I_{p_{\perp}}^{ \pm}
$$

where the quantities $I_{p_{\perp}}^{ \pm}$are given by Eq. (57).

It follows from Eq. (78) that the density of created pairs (71) and the probability of the vacuum to remain a vacuum (72) obtained with the help of the slowly varying field approximation coincide with expressions (59) and (60), respectively. In the framework of the slowly varying field approximation one can obtain expressions for the vacuum means of current density and EMT given by Eqs. (61) and (62), respectively.

\section{FINAL REMARKS}

As stated in the Introduction, until now, there were known only few exactly solvable cases in strong-field $Q E D$ with $t$-steps. In the paper we present a new case of this kind, the corresponding $t$-step is given by a time-dependent analytic asymmetric field (4). For a nonperturbative analysis of the vacuum instability generated by such an external field, we have followed the well-known general approach proposed in the works [5,12], based on the use of the corresponding exact solutions (in particular in- and outsolutions) of the Dirac equation.

One of the main and new result of the work was finding such solutions. It must be said that this problem turned out to be completely nontrivial. Unlike previously mentioned exactly solvable cases, for the asymmetric analytical field we had to apply an original method based on an analogue of Darboux transformation. With help of this method, we find solutions of the Dirac equation in the form of the differential transformation (12) of the Gaussian hypergeometric functions. Then we construct complete sets of in- and outsolutions of the Dirac equation with the asymmetric analytic field, see Sec. II.

With the help of these sets (following the above mentioned nonperturbative techniques), we have calculated exactly basic characteristics of the vacuum instability in the electric field under consideration, namely the vacuumto-vacuum transition probability $P_{\mathrm{v}}$, differential $N_{n}$ and total $N$ mean numbers of created pairs, see Sec. III. Next, we compare the obtained characteristics with the corresponding characteristics of vacuum instability in other exactly solvable cases. We analyze the dependence of the calculated quantities on the timescale parameter $\sigma$, which determines the shape of the analytic asymmetric electric field.

We note that in the case of a weak analytic asymmetric field, the obtained results are reduced to results which can be derived in the framework of a perturbation theory with respect to the external field. This, in particular, is evidenced by the expression for the differential mean numbers (32) obtained for the case of the weak field.

If the analytic asymmetric electric field is strong and the increment $\Delta W$ of the longitudinal momentum is large enough we deal with the case of a rapidly changing electric field. In this case, as it follows from Eq. (37), the differential mean numbers $N_{n}$ reach their maximum possible for fermions values $N_{n} \approx 1$, in wide ranges of the momenta $p_{x}^{\prime}$ and $\pi_{\perp}$. The width of each of these ranges is only one order less than the increment $\Delta W$, see Eq. (37). Note that this behavior is inherent to all short pulses with large potential steps belonging to exactly solvable cases, see Ref. [18]. 
As was already mentioned, among external fields of all exactly solvable cases, only the external field of the new case, considered in the present work, is given by an analytic function, which is not symmetric with respect to its maximum value. This circumstance makes it possible to analyze nonperturbatively exactly the influence of such asymmetry on effects of the vacuum instability. In particular, the influence of the asymmetry on the particle production can be seen by the example of considering the differential numbers (28). Due to this asymmetry these quantities behave differently as functions of positive and negative longitudinal momenta $p_{x}$. Apparently, a more detailed analysis of asymmetry effects should be carried out by comparing the vacuum instability in the analytic asymmetric field with the one in Sauter-like field, since the latter field is similar but differs by the presence of an asymmetric right part. In this case, it may be useful to clarify the role of the symmetry (with respect to the middle point), which has no particular physical significance, inherent in the configurations of external fields that are simplified for obtaining exact solutions.

This is evident in the further analysis. For example, when analyzing the differential mean numbers [see Eq. (40)] in the case of large $\sigma$, or equivalently in the case of the slowly varying analytic asymmetric electric field, the function $\tau$ which determines the behavior of these numbers, contains in the leading term in the denominator a third-order term in longitudinal kinetic momenta at time $t_{\max }, p_{x}^{\prime}$ [see Eq. (46)] which significantly changes the behavior of the mean differential numbers over the regions of momenta in comparison with their behavior (see Ref. [36]) in the case of symmetric Sauter-like electric field.

The exact results obtained make it possible to see clearly how the vacuum instability behaves in a slowly varying field, which, in particular, corresponds to large values of the parameter $\sigma$. In this case, it was shown (see Eq. (46) and the accompanying discussion) that, in a fairly wide range of momenta $\pi_{\perp} \ll e E_{0} \sigma, p_{x}^{\prime} \ll e E_{0} \sigma$, the differential mean numbers do not depend on $\sigma$ and coincide with ones produced by a constant electric field field, see Eq. (48). It is also shown that the total vacuum mean number of created pairs $N \approx V_{(d-1)} \rho^{\Omega}$, as well as the corresponding density $\rho^{\Omega}$ and the current density, are proportional to the increment $\Delta W$, while dominant contributions to the energymomentum tensor of created particles are proportional to the square of the increment, similar to the cases of other slowly varying fields (see Ref. [18]).

In Sec. IV, we compare the total mean number $N$ of created pairs in the regime of the slowly varying field with an estimate obtained in an universal slowly varying field approximation proposed in Ref. [34], thus demonstrating the effectiveness of the latter. We stress that the agreement with predictions of the universal slowly varying field approximation is quite expected, since in this case the density $\rho^{\Omega}$ is proportional to a large parameter, namely, the amount of "work" that the field does by generating pairs. The shape of the electric field determines the proportionality factor, see Eqs. (65) and (59). These factors are quite similar for strong electric fields, but are significantly different otherwise. It also should be noted that the cubic dependence of the differential mean numbers (46) on the longitudinal momenta $p_{x}^{\prime}$, which arises due to the asymmetry, is essential for a correct calculation of the total mean number $N$. Indeed, if for some reason, one omits the cubic term in representation (46), the resulting total mean number $N$ would be significantly different. In addition to the above, the field asymmetry affects the differential mean numbers since the leading term in the factor $\tau^{-1}$ depends on $p_{x}^{\prime 3}$. There was no such dependence for the case of the symmetric Sauter-like field, as can be seen by comparing Eq. (46) of the current work with the corresponding results in the article [18].

\section{ACKNOWLEDGMENTS}

The work is supported by Russian Science Foundation, Grant No. 19-12-00042.

\section{APPENDIX: SOME PROPERTIES OF HYPERGEOMETRIC FUNCTIONS}

The hypergeometric function $F(a, b, c ; z)=$ ${ }_{2} F_{1}(a, b, c ; z)$ (here and in what follows it is supposed that parameters $a$ and $b$ are not equal to $0,-1,-2, \ldots)$ is defined by series

$$
\begin{aligned}
F(a, b, c ; z)= & \sum_{n=0}^{+\infty} \frac{(a)_{n}(b)_{n}}{(c)_{n}} \frac{z^{n}}{n !}=\frac{\Gamma(c)}{\Gamma(a) \Gamma(b)} \\
& \times \sum_{n=0}^{+\infty} \frac{\Gamma(a+n) \Gamma(b+n)}{\Gamma(c+n)} \frac{z^{n}}{n !}, \quad|z|<1 .
\end{aligned}
$$

Note that in the solutions (16) and (17) the arguments $1-\xi^{-1}$ and $\xi^{-1}$ in the corresponding hypergeometric functions are less than unity and the series (A1) converges.

At $|z|=1$ the series (A1) converges absolutely when $\operatorname{Re}(c-a-b)>0$. The integral representation

$$
\begin{aligned}
& F(a, b, c ; z) \\
& =\frac{\Gamma(c)}{\Gamma(b) \Gamma(c-b)} \int_{0}^{1} t^{b-1}(1-t)^{c-b-1}(1-z t)^{-a} d t, \\
& \quad(\operatorname{Re} c>\operatorname{Re} b>0)
\end{aligned}
$$

gives an analytical continuation for the function $F(a, b, c ; z)$ to the complex $z$-plane with a cut along the real axis from 1 to $\infty$ (since the right-hand side is an unambiguous analytic function in the domain $|\arg (1-z)| \leq \pi)$. From the integral representation (A2) 
it is easy to see that $\lim _{z \rightarrow 0} F(a, b, c ; z)=1$. The formula for differentiating the hypergeometric function has the form:

$$
\frac{d}{d z} F(a, b, c ; z)=\frac{a b}{c} F(a+1, b+1, c+1 ; z) .
$$

It is follows from (A2) that

$$
F(a, b, c ; z)=(1-z)^{c-a-b} F(c-a, c-b, c ; z), \quad|z|<1 .
$$

Hypergeometric function can be transformed as

$$
\begin{aligned}
& F(a, b, c ; z) \\
& =\frac{\Gamma(c) \Gamma(c-a-b)}{\Gamma(c-a) \Gamma(c-b)} F(a, b, a+b-c+1 ; 1-z) \\
& \quad+(1-z)^{c-a-b} \frac{\Gamma(c) \Gamma(a+b-c)}{\Gamma(a) \Gamma(b)} \\
& \quad \times F(c-a, c-b, c-a-b+1 ; 1-z), \\
& \quad(|\arg (1-z)|<\pi),
\end{aligned}
$$

The hypergeometric equation in its general form,

$$
z(1-z) w^{\prime \prime}(z)+[c-(a+b+1) z] w^{\prime}(z)-a b w(z)=0,
$$

has three regular singular points $z=0,1, \infty$. When none of the numbers $c, c-a-b, a-b$ is integer, the general solution $w(z)$ of the hypergeometric equation (A6) can be obtained as

$$
\begin{array}{ll}
w(z)=c_{1} w_{1}(z)+c_{2} w_{2}(z), & z \rightarrow 0, \\
w(z)=c_{1} w_{3}(z)+c_{2} w_{4}(z), & z \rightarrow 1, \\
w(z)=c_{1} w_{5}(z)+c_{2} w_{6}(z), & z \rightarrow \infty
\end{array}
$$

where $c_{1}$ and $c_{2}$ are some constants, and the functions $w_{j}(z), j=1, \ldots, 6$, have the form:

$w_{1}(z)=F(a, b, c ; z)$,

$w_{2}(z)=z^{1-c} F(a-c+1, b-c+1,2-c ; z)$,

$w_{3}(z)=F(a, b, a+b+1-c ; 1-z)$,

$w_{4}(z)=(1-z)^{c-a-b} F(c-b, c-a, c-a-b+1,1-z)$,

$w_{5}(z)=z^{-a} F\left(a, a-c+1, a-b+1, z^{-1}\right)$,

$w_{6}(z)=z^{-b} F\left(b, b-c+1, b-a+1, z^{-1}\right)$.

The Kummer relations and for the hypergeometric equation [39] allow us to represent the functions $w_{1}(z)$ and $w_{2}(z)$ via the functions $w_{3}(z)$ and $w_{4}(z)$,

$$
\begin{aligned}
w_{1}(z)= & e^{i \pi\left(2 \alpha_{1}-b\right)} \frac{\Gamma\left(2\left(\alpha_{1}+1\right)-a-b\right) \Gamma(b-a+1)}{\Gamma(2-a) \Gamma\left(2 \alpha_{1}-a+1\right)} w_{4}(z) \\
& -e^{i \pi\left(2 \alpha_{1}-a\right)} \frac{\Gamma\left(2\left(\alpha_{1}+1\right)-a-b\right) \Gamma(a-b-1)}{\Gamma(1-b) \Gamma\left(2 \alpha_{1}-b\right)} w_{3}(z),
\end{aligned}
$$

$$
\begin{aligned}
w_{2}(z)= & e^{i \pi(a-1)} \frac{\Gamma\left(a+b-2 \alpha_{1}\right) \Gamma(b-a+1)}{\Gamma\left(b-2 \alpha_{1}+1\right) \Gamma(b)} w_{4}(z) \\
& +e^{i \pi b} \frac{\Gamma\left(a+b-2 \alpha_{1}\right) \Gamma(a-b-1)}{\Gamma\left(a-2 \alpha_{1}\right) \Gamma(a-1)} w_{3}(z) .
\end{aligned}
$$

[1] J. Schwinger, Phys. Rev. 82, 664 (1951).

[2] A. I. Nikishov, in Quantum Electrodynamics of Phenomena in Intense Fields, Proc. P.N. Lebedev Phys. Inst. (Nauka, Moscow, 1979), Vol. 111, p. 153.

[3] N. D. Birrell and P. C.W. Davies, Quantum Fields in Curved Space (Cambridge University Press, Cambridge, England, 1982).

[4] W. Greiner, B. Müller, and J. Rafelski, Quantum Electrodynamics of Strong Fields (Springer-Verlag, Berlin, 1985).

[5] E.S. Fradkin, D. M. Gitman, and S. M. Shvartsman, Quantum Electrodynamics with Unstable Vacuum (Springer-Verlag, Berlin, 1991).

[6] A. A. Grib, S. G. Mamaev, and V. M. Mostepanenko, Vacuum Quantum Effects in Strong Fields (Friedmann Laboratory, St. Petersburg, 1994).

[7] R. Ruffini, G. Vereshchagin, and S. S. Xue, Phys. Rep. 487, 1 (2010).
[8] G. V. Dunne, Eur. Phys. J. D 55, 327 (2009).

[9] N. Vandecasteele, A. Barreiro, M. Lazzeri, A. Bachtold, and F. Mauri, Phys. Rev. B 82, 045416 (2010).

[10] S. P. Gavrilov, D. M. Gitman, and N. Yokomizo, Phys. Rev. D 86, 125022 (2012).

[11] M. I. Katsnelson, G. E. Volovik, and M. A. Zubkov, Ann. Phys. (Amsterdam) 336, 36 (2013).

[12] D. M. Gitman, Sov. Phys. J. 19, 1309 (1976); 19, 1314 (1976); D. M. Gitman and S. P. Gavrilov, Sov. Phys. J. 20, 75 (1977); D. M. Gitman, J. Phys. A 10, 2007 (1977); E. S. Fradkin and D. M. Gitman, Fortschr. Phys. 29, 381 (1981).

[13] E. Brezin and C. Itzykson, Phys. Rev. D 2, 1191 (1970).

[14] N. B. Narozhny and A. I. Nikishov, Sov. Phys. JETP 38, 427 (1974), http://www.jetp.ras.ru/cgi-bin/e/index/e/38/3/p427? $\mathrm{a}=$ list.

[15] V. M. Mostepanenko and V. M. Frolov, Sov. J. Nucl. Phys. (USA) 19, 451 (1974). 
[16] S. P. Gavrilov and D. M. Gitman, Phys. Rev. D 93, 045002 (2016).

[17] S. P. Gavrilov and D. M. Gitman, Eur. Phys. J. C 80, 820 (2020).

[18] T. C. Adorno, S. P. Gavrilov, and D. M. Gitman, Int. J. Mod. Phys. A 32, 1750105 (2017).

[19] T. C. Adorno, S. P. Gavrilov, and D. M. Gitman, Eur. Phys. J. C 78, 1021 (2018).

[20] N. B. Narozhny and A. I. Nikishov, Yad. Fiz. 11, 1072 (1970) [Transl.Sov. J. Nucl. Phys. (USA) 11, 596 (1970)].

[21] F. Gelis and N. Tanji, Prog. Part. Nucl. Phys. 87, 1 (2016).

[22] M. Lewkowicz and B. Rosenstein, Phys. Rev. Lett. 102, 106802 (2009).

[23] B. Rosenstein, M. Lewkowicz, H. C. Kao, and Y. Korniyenko, Phys. Rev. B 81, 041416(R) (2010).

[24] H. C. Kao, M. Lewkowicz, and B. Rosenstein, Phys. Rev. B 82, 035406 (2010).

[25] N. Vandecasteele, A. Barreiro, M. Lazzeri, A. Bachtold, and F. Mauri, Phys. Rev. B 82, 045416 (2010).

[26] M. A. Zubkov, Pis'ma Zh. Eksp. Teor. Fiz. 95, 540 (2012).

[27] L. Klimchitskaya and V. M. Mostepanenko, Phys. Rev. D 87, 125011 (2013).

[28] F. Fillion-Gourdeau and S. MacLean, Phys. Rev. B 92, 035401 (2015).

[29] S. Vajna, B. Dóra, and R. Moessner, Phys. Rev. B 92, 085122 (2015).

[30] P. R. Anderson and E. Mottola, Phys. Rev. D 89, 104038 (2014).

[31] E. T. Akhmedov and F. K. Popov, J. High Energy Phys. 09 (2015) 085.
[32] C. Stahl, E. Strobel, and S. S. Xue, Phys. Rev. D 93, 025004 (2016).

[33] Ch.-M. Chen and S. P. Kim, Phys. Rev. D 101, 085014 (2020).

[34] S. P. Gavrilov and D. M. Gitman, Phys. Rev. D 95, 076013 (2017).

[35] R. Brauer and H. Weyl, Am. J. Math. 57, 425 (1935).

[36] S. P. Gavrilov and D. M. Gitman, Phys. Rev. D 53, 7162 (1996).

[37] NIST Digital Library of Mathematical Functions, edited by F. W. J. Olver et al., http://dlmf.nist.gov/, 2015-08-07 DLMF Update; Version 1.0.10.

[38] F. M. Arscott, Heun's Differential Equations, edited by A. Ronveaux (Oxford University Press, Oxford, 1995).

[39] Higher Transcendental Functions, edited by A. Erdélyi, Bateman Manuscript Project Vols. 1, 2 (McGraw-Hill, New York 1953).

[40] A. I. Nikishov, Zh. Eksp. Teor. Fiz. 57, 1210 (1969) [Transl. Sov. Phys. JETP 30, 660 (1970)], http://www.jetp.ras.ru/cgibin/e/index/e/30/4/p660?a=list.

[41] G. A. Korn and T. M. Korn, Mathematical Handbook for Scientists and Engineers: Definitions, Theorems, and Formulas for Reference and Review (Dover, New York 2000).

[42] G. Dunne and T. Hall, Phys. Rev. D 58, 105022 (1998).

[43] V. P. Gusynin and I. A. Shovkovy, Can. J. Phys. 74, 282 (1996).

[44] V. P. Gusynin and I. A. Shovkovy, J. Math. Phys. (N.Y.) 40, 5406 (1999).

[45] F. Karbstein, J. High Energy Phys. 09 (2021) 070.

[46] G. V. Dunne, in From Fields to Strings: Circumnavigating Theoretical Physics, edited by M. Shifman, A. Vainshtein, and J. Wheater (World Scientific, Singapore, 2005). 